\title{
Vitamin-B1-Vorstufe schützt Nervenfasern
}

\begin{abstract}
Für Diabetiker ist das Risiko groß, eine Neuropathie zu entwickeln. Die Vitamin-B1-Vorstufe Benfotiamin gilt zusammen mit Alpha-Liponsäure als pathogenetisch wirksamer Therapieansatz, mit dem neuropathische Schmerzen und hyperglykämiebedingte Schäden vermindert werden können.
\end{abstract}

Etwa jeder dritte Diabetiker hat eine Polyneuropathie. Die Bandbreite der möglichen Symptome reicht von Schmerzen über Parästhesien bis zu Gefühlstaubheit. Fast die Hälfte dieser Patienten habe eine verminderte Schmerzwahrnehmung, so Prof. Oliver Schnell vom Helmholtz-Zentrum in München. Die Folgen: Unerkannte Verletzungen an den Füßen zwingen nicht selten zu Amputationen.

Frühester objektiver und zuverlässiger Indikator für eine distal-symmetrische Polyneuropathie (DSPN) ist, nach Ansicht des englischen Diabetologen Prof. Solo- mon Tesfaye vom Royal Hallamshire Hospital die verminderte Nervenleitungsgeschwindigkeit. Sie ist aber meist subklinisch. Obligat in der Diabetikerbetreuung sind daher die Fußinspektion sowie Prüfung der Muskeleigenreflexe, des Vibrationsempfindens mit der Stimmgabel und des Temperaturempfindens.

Prof. Dan Ziegler vom Universitätsklinikum Düsseldorf sieht in der Optimierung des Glukosespiegels und dem Management weiterer klassischer Risikofaktoren das Fundament der Neuropathie-Therapie. Doch das reicht meistens nicht, zusätzlich sind pathogenetisch orientierte Ansätze erforderlich, um die zytotoxischen Auswirkungen der Hyperglykämie einzudämmen, wie Prof. Peter Kempler von der Universität Budapest betonte. Gut verträglich und nachweislich wirksam ist die bioverfügbare Vorstufe vom Thiamin (Vitamin B1), das Benfotiamin (milgamma protekt). „Es blockiert mehrere entscheidende Stoffwechselwege, die Hyperglykämieschäden verursachen." Experimentelle wie auch klinische Studien bestätigen den Nutzen, ergänzte Schnell.

Es ist ferner nicht ausgeschlossen, dass diese Substanz auch auf das erhöhte kardiovaskuläre Risiko der Diabetiker Einfluss hat. Es mindert die endotheliale Dysfunktion. Ergänzt werden kann die Wirkung von Benfotiamin durch das Antioxidans Alfa-Liponsäure.

Bericht: Dr. med. Jochen Aumiller, München

Quelle: Pressekonferenz beim EASD-Kongress in Berlin, 4.10.2012, Veranstalter: Wörwag Pharma

\section{Je früher eingesetzt, desto effektiver}

\author{
Beim Typ-2-Diabetes ist ein dreifaches Therapieziel anzustreben: Runter \\ mit dem Blutzucker und dem Gewicht, möglichst ohne Hypoglykämien. \\ Genau das ist mit einem GLP-1-Analogon besonders gut erreichbar.
}

Eine optimale Therapie bei Typ-2-Diabetes (T2D) sollte Prof. Werner Kern aus Ulm zufolge Folgendes bieten:

- Gute Glukosekontrolle über lange Zeit mit möglichst wenig Hypoglykämien,

- Verbesserung der Betazellfunktion,

- klinisch relevante Gewichtsabnahme,

- Entschärfung weiterer kardiovaskulärer Risikofaktoren (z.B. Hypertonie und Dyslipidämie) sowie

- einfache und flexible Therapieregime. Wie sich in den letzten Jahren zeigte, können GLP-1-basierte Behandlungsregime einem suboptimalen Management des T2D entgegensteuern. Für das humane-GLP-1-Analogon Liraglutid (Victoza ${ }^{\varpi}$ wurde in einer retrospektiven Analyse von Studien mit 1530 Diabetikern belegt, dass nach 26 Wochen mit 1,8 mg Liraglutid eine mittlere Reduktion des $\mathrm{HbA}_{1 \mathrm{c}}{ }^{-}$ Wertes im Vergleich zum Ausgangswert um 1,5\%-Punkte erreicht wird. Mit 1,2 mg Liraglutid sind es immerhin noch 1,24\%-Punkte Reduktion, mit Stagliptin aber nur 0,9\%-Punkte. Zudem erreichten mit Liraglutid signifikant mehr Patienten das $\mathrm{HbA}_{1 \mathrm{c}}$-Ziel von $\leq 7 \%$ [1]. Nach $52 \mathrm{Wo-}$ chen erreichten, verglichen mit Sitagliptin, etwa doppelt so viele Patienten mit Liraglutid 1,2 mg den kombinierten Endpunkt: $\mathrm{HbA}_{1 \mathrm{c}}<7 \%$, keine Gewichtszunahme und keine Hypoglykämien [2].

Besonders wichtig für den Erfolg der Antidiabetikatherapie scheint der Startzeitpunkt zu sein. Die besten Ergebnisse mit Liraglutid wurden erzielt bei einem $\mathrm{HbA}_{1 \mathrm{c}}$-Basiswert $<8,5 \%$, Dauer des Typ2-Diabetes <4,9 Jahre und Vortherapie mit oralem Antidiabetikum oder Ernährungsumstellung. Unter diesen Voraussetzungen erzielte das GLP-1-Analogon $(1,8 \mathrm{mg})$ in der 26 -Wochen-Analyse von 7 Phase-3-Studien die besten Ergebnisse. Bericht: Dr. Jochen Aumiller, München

\footnotetext{
1. Pratley R et al. Lancet 2010;375(9724):1447-56.
} 2. Pratley R et al. Int J Clin Pract 2011;65:397-407.

Quelle: Veranstaltung beim EASD-Kongress in Berlin, 3.10.2012, Veranstalter: Novo Nordisk.

\section{Neuer GLP-1-Agonist in Phase 3 erfolgreich}

Erste Ergebnisse dreier abgeschlossener Phase-III-Studien zu Dulaglutid hat das Unternehmen Lilly bekannt gegeben. Der lang wirksame GLP-1-RezeptorAgonist befindet sich in der klinischen Prüfung (Zulassungsstudienprogramm AWARD $^{*}$ ) für die einmal wöchentliche Gabe bei Typ-2-Diabetes. Detaillierte Ergebnisse werden in 2013/14 vorgestellt. In den drei abgeschlossenen Studien wurde der primäre Endpunkt (nicht unterlegene $\mathrm{HbA}_{1 \mathrm{c}}$-Senkung von Dulaglutid 1,5 mg vs. Vergleichssubstanz) erreicht. Beide Dulaglutid-Dosen (0,75 mg, 1,5 mg) waren bei der $\mathrm{HbA}_{1 c^{-}}$ Senkung signifikant überlegen versus Exenatid 2x/d nach 26 Wochen (AWARD-1), versus Metformin nach 26 Wochen (AWARD-3) sowie versus Sitagliptin nach 52 Wochen (AWARD-5). Die meistgenannten unerwünschten Ereignisse waren gastrointestinale.

AWARD-2 und -4, werden in den nächsten Monaten abgeschlossen. Lilly plant, Dulaglutide im Laufe des Jahres 2013 zur Zulassung einzureichen.

Quelle: Lilly Diabetes 